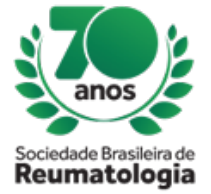

\title{
TOPICAL COLCHICINE: A POSSIBILITY OF CHEAP TREATMENT FOR LOCALIZED SCLERODERMA
}

Diana Campos Fernandino (Universidade Federal de Juiz de Fora, Juiz de Fora, MG, Brasil), Paula Reale Fernandes (Universidade Federal de Juiz de Fora, Juiz de Fora, MG, Brasil), Ludmila Godinho Figueiredo (Universidade Federal de Juiz de Fora, Juiz de Fora, MG, Brasil), Victor da Silva Coelho (Universidade Federal de Juiz de Fora, Juiz de Fora, MG, Brasil), Vania Schinzel (Universidade Federal de Juiz de Fora, Juiz de Fora, MG, Brasil)

\section{BACKGROUND}

Morphea, a subtype of localized scleroderma, is an autoimmune disease, characterized by inflammation and cutaneous fibrosis, with increased collagen deposition, especially types I and III. The current protocols propose the use of methotrexate and may be associated with systemic corticosteroids. Topical use of corticosteroids, tracolimus and calcipotriol is also described. Phototherapy is indicated for extensive cases or as an adjuvant in refractory cases. Recently the use of colchicine has been reviewed and its role in the modulation of the production of type I collagen has drawn attention to possibility of employment in the treatment of scleroderma. In view of this, we present a case with good evolution with the topical use of the medication.

\section{CASE REPORT}

YSP, 13 years old, female, in December/2018 reported 3 lesions on the back, with 2 months of evolution, treated with antihistamine, without improvement. At the physical examination, there were 3 cutaneous lesions, infiltrated, hyperchromic, sclerotic plaques: 1 plaque in the right-back region, $3 \mathrm{~cm}$ (Rodnan 2) and 2 plaques in the lumbosacral region, $7 \mathrm{~cm}$ (Rodnan 2), all without signs of atrophy. In January/2019 a cutaneous biopsy was performed and histopathology consistent with morphea. She received treatment with oral methotrexate and colchicine 5\% cream. The patient presented significant improvement of the lesions (Rodnan 0) after 4 months of treatment. Maintaining hyperchromia in the affected region.

\section{CONCLUSION}

Studies indicate that systemic colchicine plays a role in decreasing the production of collagen fibers. However, there are few papers on topical medication. The use of colchicine $5 \%$ cream can be a new possibility of treatment for a disease that causes deformities and limitations, with the great advantage of low cost and wide availability. 